\title{
Screening of hearing impairment in the newborn using the auditory response cradle
}

\author{
S M Tucker, J Bhattacharya
}

Abstract

The Auditory Response Cradle (ARC) is a fully automated microprocessor controlled machine that was designed for the hearing screening of full term neonates. In order to evaluate the ARC, 6000 babies were screened at a district maternity hospital over a period of three years. Every infant subsequently entered a three year follow up programme.

One hundred and two babies (1.7\%) failed the ARC screen (that is, they failed two ARC tests) and 20 of these were found to have some hearing impairment: in 10 it was severe (80-90 dBHL), in seven moderate (45-60 dBHL), and in three it was mild to moderate ( $<45 \mathrm{dBHL})$. In addition, of the 20 babies who failed $a$ first test and were discharged before a second could be performed, two were confirmed to have a severe hearing loss; 79 infants failing the screen were cleared on further testing, giving the ARC a false positive rate of $1.3 \%$.

On following up all 6000 infants for three years, seven children who passed the neonatal screen were subsequently found to have a hearing loss. For two babies the aetiology was unknown but for five the hearing impairment was either due to a hereditary progressive loss or definite postnatal factors. Progressive and acquired hearing losses cannot be detected at a neonatal screen and this emphasises the need for follow up screens at other stages in the child's life.

*In this long term study the ARC has been found to have a high detection rate for severe hearing loss and confirms the practical possibility of using a behavioural technique for the universal screening of hearing in neonates.

The belief that the first years of life are in many ways the most critical for the development of language, and cognitive and social/emotional skills, has stimulated increasing interest in the early detection of hearing loss. ${ }^{12}$ The early detection of hearing impairment is believed to be critical to optimal rehabilitative outcome ${ }^{3}$ but incontrovertible evidence is hard to find in the literature. Some researchers have shown that the early detection of hearing impairment and provision of hearing aids assist the acquisition of language skills. ${ }^{4}$ Other studies have shown that early fitting with hearing aids is advantageous for linguistic and cognitive development in perma- nent hearing loss. ${ }^{5} 6$ Although there is little hard scientific evidence documented, most workers would agree that there is every justification for the early detection of severely and profoundly deaf infants.

In order to reduce auditory disability successfully in the first two years of life, it is important to implement screening programmes as soon after birth as possible. ${ }^{7}$ In 1982 the Joint Committee on Infant Hearing in the United States issued a statement recommending the identification of hearing loss by 3 months of age, and no later than 6 months of age, and wherever possible the diagnostic process should be completed and habilitation begun by 6 months of age. ${ }^{8}$ Screening for hearing loss in the neonatal period also has a distinct advantage-very early detection can be achieved on a captive population.

However, there still remain at least two unresolved issues regarding the screening of newborn infants. The first relates to whether 'universal' or 'at risk' screening should be performed. As severe congenital sensorineural hearing loss is a low incidence handicap (estimated at $1-2 / 1000$ live births), the mass screening of all newborns is generally viewed as too time consuming and costly. Efforts at screening, therefore, generally focus on high risk groups such as neonatal intensive care unit (NICU) babies where the incidence of hearing loss is greater. ${ }^{910}$

The main problem with 'at risk' screening programmes is that they fall well short of identifying all children with hearing loss, as only approximately $50 \%$ of hearing impaired babies manifest one or more of the risk factors. In Pappas' study, only $46 \%$ of the children investigated would have been in the high risk category, ${ }^{11}$ and in the study of Watkin et al of a 16 year cohort less than $40 \%$ would have been identified. ${ }^{12}$ In the summary of a report on high risk screening programmes carried out in the United States and Canada it was recommended that universal screening should be the ultimate goal. ${ }^{13}$ There is therefore a strong case for the universal screening of newborn infants if this could be performed reliably and cost effectively.

A second unresolved issue regarding hearing screening of the newborn is the choice of a screening technique. Three different techniques are currently available: (i) auditory evoked potential measurements, principally brainstem response audiometry, ${ }^{14-16}$ (ii) automated behavioural assessments, ${ }^{17} 18$ and (iii) click evoked otoacoustic emissions. ${ }^{19} 20$ 
The three methods address different forms of auditory responses and provide varying degrees of information about the integrity of the acoustic system. In using auditory brainstem evoked potentials and otoacoustic emissions one is only analysing the intactness of the auditory system to the cochlea and brainstem respectively. Behavioural audiometry measures the responsivity of the long reflex pathway connecting the ear via the brainstem to the motor apparatus. Automated behavioural screeners therefore depend on the integrity of the entire auditory and motor systems and have the advantage of being able to establish that the whole auditory system is functioning correctly.

The Auditory Response Cradle (ARC) was designed and developed for normal term newborn screening and analyses objectively a neonate's behavioural responses to sound by comparing auditory responses during sound trials with spontaneous activity during 'no-sound' control trials. ${ }^{21-24}$ In order to assess the feasibility of the universal screening of neonates with the ARC for congenital severe bilateral hearing loss, a long term evaluation programme was carried out on a total of 6000 infants. The babies were screened during the neonatal period and every child was then followed up for three years. This paper presents the results of this ARC evaluation programme.

\section{Methods}

THE ARC

The ARC is a fully automatic microprocessor controlled machine that was designed and developed at Brunel University over a period of 12 years to analyse objectively a normal term neonate's behavioural responses to sound. The ARC (fig 1) consists of a trolley mounted unit comprising a pressure sensitive mattress and headrest that monitor head turn, head startle, and body activity. A transducer in a polyethylene band around the baby's abdomen (over the clothes) monitors respiratory activity. The acoustic stimulus is a high pass noise $(2 \cdot 6-4.4 \mathrm{kHz})$ of $85 \mathrm{~dB}$ sound pressure level and this can be presented binaurally

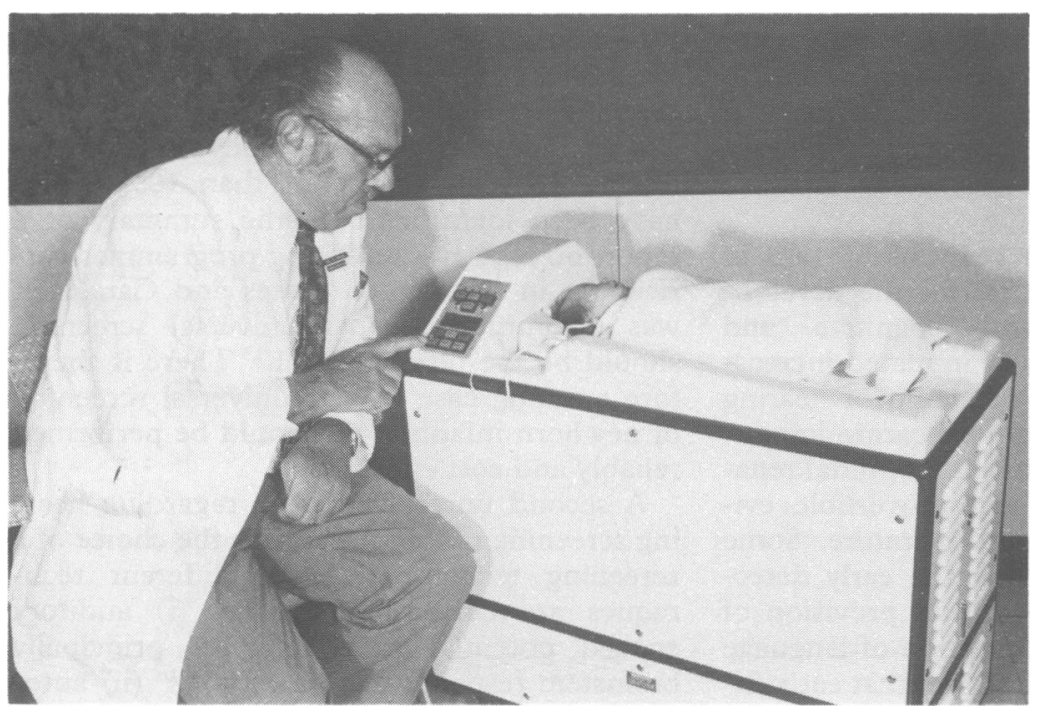

Figure 1 An infant in the $A R C$, model 6. or monaurally to the infant via close coupled ear phones.

High pass noise is used because of the prevalence of high frequency impairment among the congenitally deaf. On the presentation of the sound trials, the infant's motor and respiratory responses are detected automatically and stored in the memory of the microprocessor. The presentation of an equal number of 'no sound' (control) trials enable calculation of the probability that the baby's responses to sound are specific reactions and not just spontaneous events. When this probability exceeds $97 \%$ the baby is considered to have normal responses and is 'passed'.

\section{THE PASS/REFER DECISION}

The electronics of the ARC form a hybrid computer based around the Motorolla 6800 microprocessor. As soon as the test is initiated, the sensors are continuously examined automatically. Once the baby has become relatively quiet with identifiable respiration, the microprocessor begins acquiring data for a five second prestimulus period.

A complete screening test comprises between two and 10 trial blocks, each having two sound and two control trial elements. A trial element consists of prestimulus, stimulus and poststimulus periods, each lasting five seconds. The length of the first two periods is determined by the need for at least two complete breathing cycles for analysis. The poststimulus period, together with the presentation order within the block of 'sound/sound/ control/control' is believed to obviate habituation effects.

After the second trial block and subsequently after every further block, the scores to sound and control trials are compared using standard probability theory based on a binomial distribution. The PASS indicator on the control console automatically lights up when the scores differ by an amount giving a probability of at least $97 \%$ that the sound score could have been gained by pure chance. If this has not been achieved after 10 trial blocks have been presented to the infant, the REFER indicator is illuminated.

\section{THE EVALUATION PROGRAMME}

In order to evaluate the ARC as a universal hearing screener, it was recommended by the Department of Health that a mass screening programme with long term follow up should be carried out on a total of 6000 neonates. All these infants were to be screened while in the newborn nursery of Hillingdon Hospital (a district general hospital). Children failing the screen and found to be hearing impaired were to be managed by their local audiology centres. Children passing the neonatal screen were to be followed up by the ARC evaluation team at 7 to 9 months, 18 months, and 3 years of age in order to pick up any false negative cases.

The 6000 neonates in this study were tested over a three year period and represent $80.4 \%$ of the total number of live births (7462). It was not the aim to test every baby passing 
through the maternity unit as the ARC was only used to screen infants on three days per week. (The screening trials were carried out by two part time nursery nurses both of whom were employed for 18 hours/week.)

The neonates were screened either monaurally or binaurally on the ARC. As binaural tests were quicker they were given to babies who were not thought to be at risk for hearing impairment-that is, the majority of the 6000 infants. Neonates were tested at any time before their discharge from the maternity unit. It was found that waiting until a day or two after birth and using the period one hour after a feed to one hour before the next ensured a more settled baby. Infants from the NICU were not tested until they had reached a conceptional age of at least $\mathbf{4 2}$ weeks as the ARC was only designed for the screening of term babies. By waiting until this time, difficulties that might have arisen due to maturational factors and a weight below $2.27 \mathrm{~kg}$ were avoided. Testing was therefore only carried out on well babies.

The ARC was housed in a room at the quiet end of the antenatal ward. The test room, measuring $10 \times 17 \mathrm{ft}(3 \times 5.2 \mathrm{~m})$, was darkened with blinds and fitted with a sound attenuation door but was not otherwise sound treated. After obtaining informed consent for the test from the mother on the ward, the nursery nurse placed the polyethylene band containing the respiratory transducer around the infant's abdomen over the clothes. The baby was then taken to the test room in his/her bassinet and was swaddled to reduce the arousal state before being placed in the ARC. The earphones were gently put in place, and once the start button on the control console was depressed, a pass or refer decision was automatically made in $2-10$ minutes. The actual test time depended on the responsiveness of the infant and in this study the shortest tests obtained were for the binaural presentation of a stimuli to term babies (approximately four minutes).

A screening failure was defined as two ARC test failures. Babies failing the ARC test on two separate occasions (on two separate days) were referred for a full paediatric examination, auditory brainstem response testing (ABR), tympanometry, and acoustic reflex measurements. On ABR testing the infant was 'cleared' if wave V was observed to have a normal latency down to a stimulus level of 30 dB normative hearing level ( $\mathrm{nHL}$ ) on each ear.

When every child initially tested at birth reached the age of 7 months (with the exception of those already confirmed as hearing impaired who were followed up separately), distraction testing was carried out by the local child health clinics. All test results were sent back to the ARC evaluation team. If the child did not attend the clinic screen a questionnaire was sent to the mother with questions about the baby's babble, awareness of sounds, and history of ear infections. Any suspicion of hearing loss arising from the returned form resulted in recall for distraction testing employing both the standard stimuli used in the clinics and warble tones of $500 \mathrm{~Hz}$ to 4 $\mathrm{kHZ}$. The child was cleared if responses were obtained to all stimuli at a minimal level of 30 dBA on both sides (sound level measured in free field, that is room setting).

The subsequent follow up at 18 months of age took the form of a questionnaire which was sent to the mother of every child screened at birth except those already confirmed as hearing impaired. Children with unsatisfactory replies to the hearing, balance, and vocabulary questions were recalled for distraction testing, tympanometry, and acoustic reflex measurements. Stimuli employed included the cup and spoon, sibilant 'ss', high frequency rattle, voicing at low frequencies, and warble tones. Visual reinforcement audiometry was also employed when necessary and the child was cleared if he responded to all test stimuli at a minimal level of $30 \mathrm{dBA}$ on both sides.

The final follow up at 3 years of age took the form of a further questionnaire. Children with unsatisfactory replies to the hearing, balance, and speech questions were recalled for pure tone headphone audiometry, tympanometry, acoustic reflex measurements, a speech discrimination test, and otoscopy. The child was cleared as having hearing within normal limits if his auditory threshold was less than $20 \mathrm{dBHL}$ for $500 \mathrm{~Hz}$ to $4 \mathrm{kHz}$ and if the audiogram was supported by the speech discrimination test (with $100 \%$ discrimination being obtained at minimal level of $35 \mathrm{dBA}$ ).

Audiological assessments on children recalled during the three year follow up programme were carried out in an anaechoic chamber at Brunel University. Test results were sent to the general practitioner, consultant paediatrician, ear, nose, and throat surgeon, and district community health team. At all stages of the three year evaluation programme the attendance of children recalled was $100 \%$ as transport was provided whenever necessary.

Results

The results of the long term evaluation of the ARC are shown in tables 1 to 4 . Table 1 gives

Table 1 Results of ARC trials

\begin{tabular}{ll}
\hline No of neonates tested & 6000 \\
Failed first ARC test & 489 (8.1\%) \\
Passed on retest & 367 \\
Could not be retested on the ARC: & 20 \\
1 severe bilateral S/N loss (a) & \\
1 severe bilateral high frequency & \\
S/N loss (b) & \\
15 cleared & $102 \quad(1 \cdot 7 \%)$ \\
3 lost to follow up (1 died) & \\
Failed first and second ARC tests: & \\
7 severe bilateral S/N losses \\
1 severe unilateral S/N loss \\
1 severe bilateral S/N loss \\
with conductive overlay \\
2 moderate unilateral S/N losses (c, d) \\
1 mild/moderate bilateral S/N loss \\
5 middle ear pathologies (SOM) \\
(moderate bilateral conductive losses) \\
2 ABR abnormalities \\
(mild/mioderate bilateral S/N losses) \\
1 microcephalic \\
1 spastic quadriplegic \\
(severe bilateral S/N loss) \\
2 lost to follow up (1 died) \\
79 cleared \\
\hline (false positive rate =
\end{tabular}


the results of the screening trials during the neonatal stage. Tables 2, 3, and 4 give the results of the follow up programmes at 7 to 9 months, 18 months, and 3 years respectively.

\section{RESULTS OF ARC TRIALS}

Of the original 6000 neonates screened, 489 $(8 \cdot 1 \%)$ failed the first test but this figure was reduced to $102(1 \cdot 7 \%)$ after the second test. Seventy nine of the 102 babies referred for follow up by ABR and impedance testing were subsequently cleared, giving a false positive rate of $1 \cdot 3 \%$.

\section{First time failures}

Twenty newborn babies who failed the first ARC test were discharged from hospital before they could be tested again (table 1). One child (a) returned three weeks later and was found to have serous otitis media which was treated medically. He was subsequently tested elsewhere with electrocochleography and no action potential was observed; a severe bilateral sensorineural hearing loss was diagnosed.

The second child (b) did not return for a repeat ARC test and was lost to follow up for some time. He was eventually traced at 3 years of age when the questionnaire was returned with a comment about poor speech development. The family had moved out of the area and results from his local audiology clinic showed him to have a severe high frequency hearing loss. Of the remaining 18 infants who did not attend for retest, 15 were cleared at subsequent screens and three were lost to follow up-one died and two moved with no forwarding address.

\section{Second time failures}

Sensorineural losses - The failures at the second test (table 1) included nine babies with a severe sensorineural loss (eight bilateral, one unilateral), two with a moderate sensorineural loss (both unilateral), and one with a mild to moderate sensorineural loss (bilateral). The ABR threshold for those with severe losses was equal to or greater than $80 \mathrm{dBnHL}$. All eight children with severe bilateral sensorineural losses were issued with hearing aids.

Two children (c, d) had abnormal auditory brainstem responses on one side and no detectable responses on the other up to a stimulus level of $80 \mathrm{dBnHL}$. They subsequently attended and passed the 7 to 9 month clinic hearing screen but were nevertheless recalled for further assessment because of the ABR/distraction test disparity. Normal middle ear function was obtained on impedance testing for both children, and on distraction testing they were found to have a behavioural threshold of $30 \mathrm{dBA}$ for all frequencies on the good ear and a moderate sensorineural hearing loss on the other ear. Finally, one baby was found to have a mild to moderate bilateral sensorineural loss with a threshold of approximately 40-45 dB.

Middle ear pathologies-The incidence of otitis media in a normal population of newborn infants is uncertain, inasmuch as the case material in the reports of different authors is not homogeneous. ${ }^{25}$ In this ARC evaluation study a total of five neonates were picked up for whom subsequent testing showed a moderate bilateral hearing loss (threshold range 45-60 dBHL) which was found by tympanometry and acoustic reflex testing to be due to serous otitis media. It is not possible to know how many other babies in this study had otitis media during the neonatal period as the ARC was not designed for the detection of mild hearing loss.

Brainstem latency and rate abnormalties-Two brainstem latency and rate abnormalities were detected. One infant was born 6 weeks preterm, was severely jaundiced, and had been in intensive care for a week as a result of episodes of apnoea. No brainstem responses could be detected on the right ear up to a stimulus intensity of $80 \mathrm{dBnHL}$, and tympanometry showed middle ear dysfunction (serous otitis media). The second infant was found to have abnormal brainstem responses on both ears. On distraction testing at 8 months poorly localised 45-50 dBA responses were obtained and tympanometry showed bilateral middle ear dysfunction. As it was unclear whether the poor localisation was due to developmental immaturity or a sensorineural loss with conductive overlay, the child was retested at a later date. Both children were confirmed to have a mild to moderate $(<45$ $\mathrm{dBHL}$ ) sensorineural loss.

Other abnormalities - One of the babies who failed the ARC test was subsequently found to be microcephalic. There was no indication of any high risk factors and on ABR testing normal latencies were obtained for wave $V$ down to a stimulus level of $40 \mathrm{dBnHL}$. During ABR testing the child's electroencephalographic trace was continuously monitored. In the case of this baby it appeared abnormal and after a full paediatric assessment he was diagnosed as microcephalic. Another infant who failed the ARC test was resuscitated after apnoea and asystole: on ABR testing poor responses were

Table 2 Results of 7-9 month clinic follow up

No of children now 7 months old (18 died) 5982 Passed the clinic screen:

These included:

$4917(82 \cdot 2 \%)$

2 moderate unilateral $S \mathrm{~N}$ losses (failed the ARC screen) (c, d)

1 severe bilateral S/N loss

1 severe bilateral S/N loss

(passed the ARC screen,
aetiology unknown) (e)

Failed the clinic screen:

1 severe bilateral S/N loss

(failed the ARC screen) (a)

1 severe bilateral S/N loss (passed the ARC screen

aetiology unknown) (f)

1 severe bilateral $\mathbf{S} / \mathrm{N}$ loss
(failed the ARC screen) (g)

1 (failed the ARC screen) (g)

ARC screen, progressive hereditary S/N loss confirmed) (h)

0 cleared -5 middle ear pathologies (SOM)

Not tested at this screen

Less number of replies to questionnaire and number already confirmed as hearing impaired

Loss to follow up at this stage

SAN, sensorineural; SOM, serous otitis media 
obtained for both ears. The child was subsequently diagnosed as having spastic quadriplegia with a severe bilateral sensorineural loss.

\section{FOLLOW UP AT 7 TO 9 MONTHS OF AGE}

As shown in table 2, 18 babies had died before reaching the age of 7 months. Of the 4917 babies who attended and passed the 7-9 month screen, three infants actually had some degree of hearing impairment. Two of these babies (c, d) had previously failed the ARC screen and ABR follow up. They were recalled for further assessment and were found to have a behavioural response threshold of $30 \mathrm{dBA}$ for all frequencies on one side and a moderate sensorineural loss on the other $(60 \mathrm{dBA}$ at $500 \mathrm{~Hz}, 1 \mathrm{kHz}$, and $2 \mathrm{kHz}$; $90 \mathrm{dBA}$ at $4 \mathrm{kHz}$ ). The third child (e) had passed both the ARC screen and the 7 to 9 month clinic screen. He was subsequently confirmed to have a severe bilateral sensorineural loss but the aetiology is unknown.

Fourteen babies failed the clinic hearing screen. This figure is very low as the child health clinics 'passed' all babies initially failing due to otitis media if the middle ear dysfunction had resolved on subsequent testing. The ARC evaluation team did not have the authority to ensure that only two distraction tests were carried out on each child.

Two of the 14 babies $(\mathrm{a}, \mathrm{g})$ failing the clinic screen had previously failed the ARC screen and had already been confirmed to be hearing impaired. As previously mentioned, babies with confirmed hearing loss were not entered into the 7-9 month, 18 month, and 3 year follow up programmes. However, in the case of babies a, $g$, c, and $d$ the child health clinic requested them to attend the 7 to 9 month screen by mistake.

Twelve of the 14 babies failing the clinic screen had passed the ARC screen. One of these 12 (h) was tested by electrocochleography at 4 months of age as there was a history of progressive hearing loss in the family and, although the baby's neonatal responses were fine, there was now some concern over the hearing. A severe bilateral sensorineural loss was confirmed. One child ( $f$ ) failed the first ARC test and passed on retest; he was subsequently found to have a severe bilateral sensorineural hearing loss with unknown aetiology. The remaining 10 babies passed when tested again; five of them had otitis media.

There were no 7 to 9 month screening results available for 1051 children (17.6\%).

Table 3 Results of 18 month follow up programme

\begin{tabular}{ll}
\hline No of questionnaires sent & 5565 \\
No returned & 4164 (74.8\%) \\
Questionnaires returned, with satisfactory & \\
replies to the hearing, balance, and & 4068 \\
vocabulary questions & 96 \\
Babies with unsatisfactory questionnaires: & 96 \\
56 cleared & \\
35 middle ear pathologies (SOM) & \\
5 S/N losses & \\
1 cytomegalovirus infection & meningitis with hydrocephalus \\
1 Jervell and Lange-Nielsen syndrome & \\
1 unknown aetiology (e) &
\end{tabular}

S/N, sensorineural; SOM, serous otitis media.
This category consisted of babies who had already been diagnosed as hearing impaired at the neonatal screen, and babies who were lost to follow up for reasons of refusing the test, non-attendance, moving with no forwarding address, moving out of the follow up area, and adoption. By means of a questionnaire, as already mentioned, and through an intensive search via the family doctor, the community health service, and local housing departments, all but $59(0.99 \%)$ children were eventually traced.

FOLLOW UP AT 18 MONTHS OF AGE

A total of 5565 and not 6000 questionnaires were sent out at 18 months at this stage of the evaluation programme (table 3 ). There were three reasons for this: (i) questionnaires were not sent to families whose child were already diagnosed as hearing impaired, (ii) 18 children had died by the time of this follow up, and (iii) a number of families had moved with no forwarding address.

Of the questionnaires sent out, 4164 replies were received giving a $74.8 \%$ return rate. Of the questionnaires returned, 4068 gave satisfactory replies to the hearing, balance, and vocabulary questions. Ninety six children were recalled for further testing: 56 were cleared and 35 were found to have middle ear dysfunction (serous otitis media) and were referred through the consultant paediatrician and senior clinical medical officer for appropriate management.

Five children who initially passed the ARC test during the neonatal stage were found to have a sensorineural loss at this follow up. One baby with cytomegalovirus infection had his ARC test result (a 'pass') confirmed on the same day with $A B R$ testing. At three months of age the ABR threshold was $45 \mathrm{dBnHL}$ for the right ear and $65 \mathrm{dBnHL}$ for the left. At 11 months the threshold had increased by $15 \mathrm{~dB}$ on both sides. This rapidly progressing sensorineural loss is a well accepted feature of virus invasion and was carefully monitored in this child.

The second child was perfectly healthy and normal at the time of the ARC test. He subsequently contracted meningitis, had a respiratory arrest at 9 weeks, and is now severely retarded with hydrocephalus and a ventricular shunt. On ABR testing, normal responses were obtained for both ears down to 20 $\mathrm{dBnHL}$. However, distraction testing suggested a central loss.

The third baby showed perfectly normal responses to sound during the neonatal period. At 6 months of age he developed meningitis with subsequent hydrocephalus and at 18 months was confirmed retarded with a severe bilateral sensorineural loss.

The fourth child also showed normal responses to sound during the neonatal period but at 8 months of age Jervell and LangeNielsen syndrome was diagnosed and, on distraction testing, only responses to low frequencies at $90 \mathrm{dBA}$ could be obtained. By 15 months of age no response could be obtained at stimulus levels up to $100 \mathrm{dBA}$. 
Table 4 Results of 3 year follow up programme

\begin{tabular}{ll}
\hline No of questionnaires sent & 5589 \\
No returned & 3737 (66.9\%) \\
Questionnaires returned, with satisfactory & \\
replies to the hearing, balance, and & 3389 \\
language questions & 348 \\
Children with unsatisfactory replies: & \\
219 cleared & \\
128 middle ear patholgies (SOM) & \\
1 bilateral high frequency S/N loss (b) & \\
\hline
\end{tabular}

The final baby (e) passed both the ARC screen and the 7 to 9 month clinic screen. At 18 months he was confirmed to have a severe bilateral sensorineural loss. The aetiology was unknown.

\section{FOLLOW UP AT 3 YEARS OF AGE}

A total of 5589 three year questionnaires were sent out at this stage of the evaluation programme and $3737(66.9 \%)$ were returned (see table 4). As a result of the 348 questionnaires returned with unsatisfactory replies, 347 children were recalled: 219 of these children were cleared and 128 were found to have middle ear dysfunction (serous otitis media).

The remaining child (b) had an audiogram carried out by his local audiology clinic and results indicated a severe bilateral high frequency hearing loss. This child had failed the ARC test once in the neonatal period (see table 1) and had not returned for a retest. As the family had moved outside the health district no information was obtainable on the 7 to 9 month clinic screen and the parents had not replied to the 18 month questionnaire. The child was eventually picked up via the three year questionnaire because of poor speech development.

\section{Discussion}

RESULTS OF THE ARC EVALUATION PROGRAMME Very large numbers of babies are needed to validate fully a screening device. For this reason it was recommended by the Department of Health that a total of 6000 newborn infants should be screened with the ARC and each child should be followed up for three years.

The ARC was designed for the detection of moderate and severe hearing impairment. As can be seen from table 1 , of the 102 neonates failing the ARC tests, a total of 20 babies were detected as having some hearing impairment-10 severe (80-90 dBHL), seven moderate (45-60 dBHL), and three mild to moderate $(<45 \mathrm{dBHL})$. In addition, two of the 20 babies who failed a first ARC test but could not be retested were found to have a severe bilateral sensorineural hearing loss.

Estimates for the incidence of congenital hearing loss do vary. A study in the European Community gives the prevalence for losses over $50 \mathrm{~dB}$ in the better ear at $500 \mathrm{~Hz}, 1 \mathrm{kHz}$, and $2 \mathrm{kHz}$ (in 8 year olds) as $1 / 1000 .^{26}$ The prevalence in a Danish study was $1 \cdot 4 / 1000,10$ and in a study carried out in Jerusalem, $1 \cdot 7 / 1000$ for children over 5 years of age. ${ }^{27} \mathrm{~A}$ common working estimate for the universal prevalence of severe congenital hearing loss is $1-2 / 1000$ live births and the incidence of any degree of hearing loss ${ }^{28}$ may be as high as $5 / 1000$.
As can be seen from table 1 , a total of 12 babies were found to have a severe hearing loss-11 bilateral, one unilateral. However, for two of these babies only one ARC test was administered as they were discharged from hospital before they could be retested. It is therefore not possible to know if they would have failed a second ARC test during the neonatal period. In this particular population of 6000 neonates the incidence of severe congenital bilateral hearing impairment appears to be $1 \cdot 5 / 1000$ (that is nine cases).

Of the 6000 neonates, 79 of the 102 who failed two ARC tests were found to have normal hearing on further testing and were subsequently cleared. This false positive rate of $1.3 \%$ is acceptably low for a screening technique and compares well with the figure of $1.5 \%$ obtained by Shepard in which the ARC was used in a modified mass screening programme. $^{29}$ The advantage of a low false positive rate is that audiological assessment facilities do not become swamped with babies requiring further testing. Unless they can cope with the additional referrals within an acceptably short time period, one only defeats the purpose of early detection. Hearing impairment should be confirmed and habilitation programmes initiated as soon as possible after the neonatal screen.

The exact number of children who lose their hearing during the first year or so of life, due to progressive or acquired losses, is unknown. One year into the ARC evaluation trials, three children who had initially passed the screen during the neonatal period were found to have a severe bilateral hearing loss (babies e, $f$, and $h$ from table 2). For two of these children (e and $f$ ) the aetiology was unknown. For the third (h), the hearing impairment was due to a hereditary progressive loss. By the time every one of the 6000 infants had been followed up for three years, another four children were found to have hearing impairment-one from cytomegalovirus infection, two from meningitis with hydrocephalus, and on with the Jervell and Lange-Nielsen syndrome (table 3 ).

These results indicate that for five of the seven children who passed the ARC screen and were subsequently found to have a hearing loss, the loss was due to either hereditary progressive or definite postnatal factors. For this reason the 'true' number of false negative cases was possibly two and not seven. The detection of children with hearing loss subsequent to the neonatal screen highlights the importance of running screening programmes at other stages in the child's life. Progressive hearing loss and hearing impairment due to postnatal factors cannot be detected in the newborn period and children need to be followed up carefully as there will always be hearing losses that do not become evident until months or even years after birth.

In this study, automated behavioural screening with the ARC has been shown to be able to provide an early, non-invasive method of detecting hearing impairment. The evaluation programme confirms the practical possi- 
bility of using a behavioural technique for the universal screening of newborn babies.

\section{FOLLOW UP LOSSES}

During the neonatal period only five of the 6000 infants screened were lost to follow up: two babies died and three moved abroad without trace. After the neonatal period the loss to follow up increased considerably from $0.99 \%$ at 7 to 9 months to $25 \cdot 2 \%$ at 18 months and $33.1 \%$ at 3 years. The only reason why follow up losses were as low as $0.99 \%$ at 7 to 9 months was because an intensive programme was carried out to trace the children via family doctor, the community health service, and local housing departments. This was extremely time consuming and would not have been possible outside a research study.

The follow up of a child subsequent to neonatal screening is one of the most difficult parts of a screening programme. This is especially so in urban metropolitan areas such as this particular district where socioeconomic and ethnic division often make recall for even routine paediatric care difficult. Also, the number of families moving with no forwarding address was particularly high and the three year loss to follow up of $33 \cdot 1 \%$ was almost entirely because of this.

This particular study has incorporated a much more intensive follow up programme than other studies where newborn infants have only been followed up for a few months or a year. In order to investigate whether there were in fact any false negative cases in the group of children lost to follow up, a major study is currently being carried out involving the educational authorities and the community health services in a number of districts to try to trace these children. The aim is to reduce the present follow up loss to negligible proportions.

Follow up losses are inevitable in studies with long term evaluation programmes (despite intensive efforts to trace all children). The increasingly greater loss to follow up with time in this study reinforces the advantage of screening when the largest proportion of the population is available-that is, in the newborn nursery.

\section{STATISTICAL PURITY OF THE PASS/REFER DECISION}

The ARC uses pass/refer decision criteria which do not vary with the number of sound or control trials given. Davis showed that the statistical criteria upon which the pass/refer decision is made should take into account the varying number of trials presented to the infant. ${ }^{30}$ However, the results in this ARC evaluation study show that the fixed criteria, for all their limitations, have correctly identified 20 babies with some hearing impairment and have only failed to identify two (if infants with acquired and progressive hearing loss are excluded).

As a separate study, over a two year period all full term neonates who required 20 or more trials to pass the ARC screen were followed up with brainstem evoked response audiometry. None of these children were found to have abnormal waveform morphology or latency intensity function. In view of these findings it is concluded that although modifications of the decision criteria could improve the statistical purity, the screening test results are unlikely to be altered with a term population.

\section{MONAURAL VERSUS BINAURAL SCREENING}

In this ARC evaluation programme the shortest test times were obtained by presenting the sound stimulus binaurally. For this reason binaural tests were given to babies who were not thought to be at risk for hearing impairment-that is, the majority of the 6000 infants. Some workers feel that if one tests binaurally and accepts that unilateral hearing losses will have a lower probability of being detected, this could lead to false assurances being offered to parents about the integrity of their child's hearing. In the study carried out by McCormick et al it was concluded that each ear should be tested separately. ${ }^{31}$ This not only affords a greater possibility of detecting a unilateral condition, but gives a more thorough test for a bilateral condition.

In this ARC study it was felt that the policy of giving babies not at risk for hearing loss a binaural test did not affect the detection rate, as approximately half of the hearing impaired babies detected by the ARC were found to be in this population. These results highlight the value of a universal hearing screening programme.

The principal aim of this study was to assess the ARC's ability to detect severe bilateral hearing loss. Screening programmes may well eventually aim to detect unilateral hearing loss as data on the behavioural and linguistic manifestations of such losses indicate that these children experience more problems than previously supposed. . $^{32}$

\section{PRACTICAL ASPECTS OF SCREENING \\ WITH THE ARC}

As already mentioned, the ARC has the advantage over electrophysiological and otoacoustic emissions techniques of assessing the integrity of a neonate's entire auditory pathway rather than just a part of it. It also has the advantages of being totally non-invasive and very acceptable to parents, of being easy to use with a simple pass or refer result, and of having a short test time when used with healthy babies. Short test times were also reported for the ARC in the modified mass screening programme carried out by Shepard. ${ }^{29}$ Here, by not testing NICU babies until they were well, test times of four to 10 minutes were obtained on the binaural presentation of stimuli.

The time taken to test each neonate is an important consideration for any screening programme. Stevens et al found that screening with an evoked otoacoustic emissions technique produced a mean test time of $12 \cdot 1$ minutes as compared with a mean of 21.0 minutes with electrophysiological screening (ABR). ${ }^{33}$ However, direct comparisons between the ARC and these two techniques 
are difficult to make as the former was used in a universal screening programme and the study of Stevens et al concentrated on babies taken largely from a neonatal intensive care unit. Also, it is difficult to know how much time was required in the latter study for affixing electrodes, data analysis, and other practical or administrative aspects associated with screening programmes.

In the present study, although the actual ARC test only lasted several minutes, half an hour was allocated per baby in order to allow for all the other necessary duties-for example, transporting the baby to and from the test room, preparing for the test, recording patient data and test results, and organising the management of test referrals.

\section{THE COSTS INCURRED IN A MASS}

SCREENING PROGRAMME

The costs of newborn screening will vary according to the type of programme implemented. It has been felt in the past that regardless of the method used, the savings to society through early identification of hearing loss far outweigh the cost. However, today much more stringent costings are being carried out before implementing hearing screening programmes.

In this study the ARC was only used for hearing screening on three days a week and the two nursery nurses who performed the tests were employed for 18 hours a week. As there is such a rapid turnover in maternity wards today, in order to screen every newborn baby a mass screening programme would need to be in operation for five days a week. Two full time nursery nurses would be able to cover a delivery rate of 3000 a year and would ensure that a continuous screening service could be offered. Also, the employment of a part time clerk would relieve the nursery nurses of much of the record keeping and other associated paperwork that would be generated.

When implementing neonatal screening services it is very important to ensure that there is access to a well staffed audiology department so that 'screening failures' can be referred promptly for further diagnostic testing. The cost of referring these babies for further diagnostic assessment would be additional to that of the equipment and staff at the screening stage. In this study the ARC was found to have a failure rate of $8 \cdot 1 \%$ after one test and $1.7 \%$ after two. By defining the overall screen failure as two test failures, screening with the ARC has been a very cost effective method of detecting hearing loss in the newborn infant. For this reason since the completion of the evaluation programme on the 6000 neonates, the ARC has continued to be used in the maternity unit for the early detection of hearing impairment.

(Screening trials in this study were carried out using the ARC model 6 . This particular model is no longer being manufactured. By redesigning the ARC6 it has been possible to develop a new machine, the PARC, which is the size and shape of a large briefcase (fig 2)

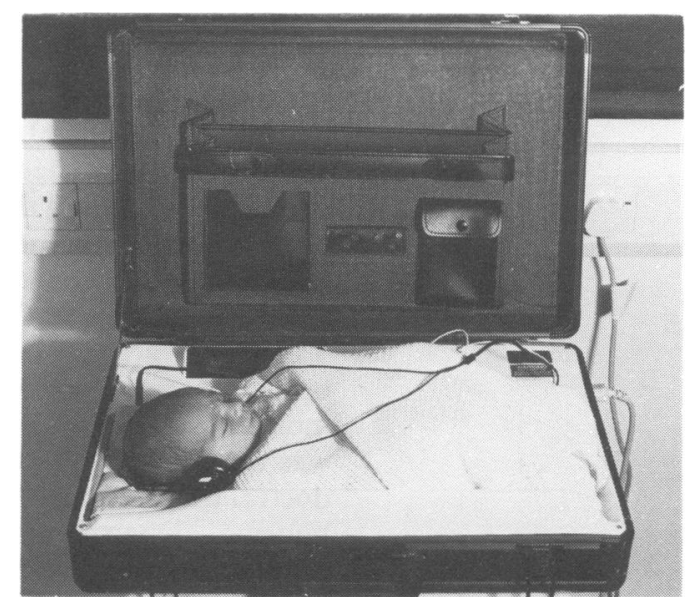

Figure 2 The PARC machine, the size of a briefcase, with the infant wearing binaural earphones.

rather than that of a trolley mounted unit. In redesigning the machine it has been possible to reduce the purchase cost to approximately £2000.)

\section{Conclusion}

The main purpose of screening for hearing impairment in newborn infants is the early detection of severe and profound bilateral sensorineural hearing loss. In this long term evaluation study of 6000 neonates, the ARC was found to have a high detection rate for this level of hearing impairment with 10 such cases having been detected after two ARC test failures. Ten babies with less severe hearing loss were also picked up. In addition, of 20 neonates failing a first ARC test who could not be retested on the ARC, two babies were found to have a severe bilateral sensorineural loss via other means.

This evaluation study of the ARC has incorporated particularly intensive follow up procedures in order that the number of false positive and false negative cases could be established as accurately as possible. A low false positive rate was obtained and this was probably because testing was only carried out on well babies. If the five children found to have hereditary progressive or acquired hearing loss are excluded, the 'true' number of false negative cases found during the three year follow up programme appears to be two. The detection of children with progressive and acquired hearing loss highlights the importance of running good follow up programmes in conjunction with any neonatal screen.

We would like to express special thanks to the nursery nurses (especially Mrs Wendy Smith) who performed all the ARC tests, and to Mr Bill Shambrook for the technical modifications of the ARC during its recent development period. (Details of the PARC may be obtained from Dr S Tucker.)

1 Marlowe JA. Hearing in infancy: the development of auditory skills and the audiological evaluation. Semin Speech Lang Hear 1982;3:28-44.

2 Parving A. Early detection and identification of congenital/early acquired hearing disability. Who takes the initiative? Int f Pediatr Otorhinolaryngol 1984;7:107-17.

3 Mahon W. Hearing care for infants and children-issues in identification and management. Hearing foumal 1987; 40(9):7-11. 
4 Greenstein JM, Greenstein BB, McConville $\mathbf{K}$ Mother-infant communication and language acquisition in deaf infants. New York: Lexington School for the Deaf, 1976.

5 Markides A. Age at fitting hearing aids and speech intelligibility. Br $\mathcal{Y}$ Audiol 1986;20:165-7.

6 Guinagh BJ, Jester RE. Long term effects of infant stimulation programs. Adv Behav Pediatr 1981;2:81-110.

7 Downs MP. Auditory screening. Otolaryngol Clin North Am 1978;11:611-29.

8 Joint Committee on Infant Hearing. Position statement. $f$ Pediatr 1982;70:496.

9 Abramovich SJ, Gregory S, Slemeck M, Stewart A. Hearing loss in very low birth-weight infants treated with neonatal intensive care. Arch Dis Child 1979;54:421-6.

10 Parving $A$. Hearing disorders in childhood: some procedures for detection, identification and diagnostic evaluation. Int $f$ Pediatr Otorhinolaryngol 1985;9:31-57.

11 Pappas DG. A study of the high risk registry for sensorineural hearing impairment. Head Neck Surg 1983;91:41-4.

12 Watkin PM, Baldwin M, Laoide S. Parental suspicion and identification of hearing impairment. Arch Dis Child identification of

13 Mahoney T. High risk screening of large general populations. Seminars in Hearing 1982;5:25-37.

14 Flood LM, Fraser JG, Conway MJ, Stewart A. The assessment of hearing in infancy using the post-auricular myogenic response. Br Y Audiol 1982;16:211-4.

15 Salamy A, Amochaev A, Somerville G. The IHAF screening device. Hearing Instruments 1983;34(6):16, 18, 47.

16 Peters JG. An automated infant screener using advanced evoked response technology. Hearing foumal 1986;39: 25-30.

17 McFarland WH, Blair-Simmons F, Jones FR. An automated hearing screening technique for newborns. I Speech Hear Disond 1980;45:495.

18 Bennett MJ, Wade HK. Automated newborn screening using the auditory response cradle. In: Taylor IG, Markides A, eds. Disonders of auditory function III. London: Academic Press, 1980:59.

19 Bray P, Kemp P. An advanced cochlear echo technique suitable for infant screening. $B r f$ Audiol 1987;21: 191-204.

20 Stevens JC, Webb HD, Hutchinson J, Connell J, Smith MF, Buffin JT. Evaluation of click-evoked oto-acoustic emissions in the newborn. Br F Audiol 1991;25:11-14.

21 Bennett MJ. Trials with the auditory response cradle I: neonatal responses to auditory stimuli. $B r \mathcal{F}$ Audiol 1979; neonatal respo

22 Bennett MJ, Lawrence RJ. Trials with the auditory response cradle II: the neonatal respiratory response to an response cradle II: the neonatal respiratory res

23 Bennett MJ. Trials with the auditory response cradle III: head turns and startles as auditory responses in the neonate. Br ₹ Audiol 1980;14:122-31.

24 Bhattacharya J, Bennett MJ, Tucker SM. Long term follow-up of newborns tested with the auditory response cradle. Arch Dis Child 1984;59:504-11.

25 Pestalozza G. Otitis media in newborn infants. Int $\mathcal{f}$ Pediatr Otorhinolaryngol 1984;8:109-24.

26 Martin JAM, Bentzen O, Colley JRT, et al. Childhood deafness in the European community. Scand Audiol 1981;10:165-74.

27 Feinmesser M, Tell L, Levi $H$. Follow-up of 40,000 infants screened for hearing deficit. Audiology 1982;21:197-203

28 Carrel RE: Epidemiology of hearing loss. In: Gerber SE, ed. Audiometry in infancy. New York: Grune and Stratton, 1977:3-16.

29 Shepard NT. Newborn hearing screening using the LincoBennett auditory response cradle: a pilot study. Ear Hear 1983;4:5-10.

30 Davis A. Detecting hearing impairment in neonates-the statistical decision criterion for the auditory response cradle. Br $\mathcal{F}$ Audiol 1984;18:163-8.

31 McCormick B, Curnock DA, Spavins F. Auditory screening of special care neonates using the auditory response cradle. Arch Dis Child 1984;59:1168-72.

32 Bess FH. Children with a unilateral hearing loss. foumal of Paediatric Audiology 1982;15:131-44.

33 Stevens JC, Webb HD, Hutchinson J, Connell J, Smith MF, Buffin JT. Click evoked otoacoustic emissions compared with brain stem electric response. Arch Dis Child 1989;64:1105-11.

Pertussis (4): prophylactic erythromycin

An outbreak of pertussis happended in Phoenix, Arizona in 1988 (Mary Ann Sprauer and colleagues, American Fournal of Diseases of Children 1992;146:177-81). Forty percent of cases were over 7 years old. The authors examined particularly the possibly protective effect of giving erythromycin to patients and contacts. Thirty seven households with 189 members were studied and secondary spread of the disease occurred in 17 households. Most of the primary cases had been treated with erythromycin (all of those in households in which there was no secondary spread and $76 \%$ of those in households with secondary spread). Treatment was started earlier, however, in the no spread households (median $11 v 21$ days).

There was no significant difference between the two groups (spread and no spread) as regards the proportion of household contacts given prophylactic erythromycin or the duration of prophylaxis but again there was a significant delay in giving the erythromycin to contacts in the households where secondary spread occurred ( $22 v 16$ days; $\mathrm{p}=0.0008)$.

The authors conclude that the early administration of erythromycin to cases and contacts is effective in preventing the spread of pertussis. They recommended a 14 day course. Their data do not fill me with enthusiasm but it is a way of attempting to stop the spread of the disease. 